\title{
Images
}

\section{Hookworm-related cutaneous larva migrans}

\author{
Eric B. Tomich, DO; Tristan Knutson, MD; Lindsay Welsh
}

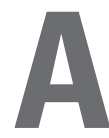

58-year-old man presented to the emergency department of a military hospital in Iraq with an expanding pruritic lesion on the sole of his right foot (Fig. 1). He denied having any systemic symptoms. The patient first noticed the lesion 3 days before his presentation and had been applying bacitracin ointment to the area with no change. Of note, he had recently returned from a 2-week vacation to the Philippines where he reported having been barefoot most of the time.

A diagnosis of hookworm-related cutaneous larva migrans (CLM) was given. Cutaneous larva migrans is the most common travel-associated skin disease of tropical origin. "Also referred to as a "creeping eruption," CLM is a generic term used to describe a variety of conditions in which there is daily progression, up to several centimetres, of a slightly elevated, erythematous rash in a linear or serpiginous pattern. ${ }^{2}$ Hookworm-related CLM is acquired from direct contact of exposed skin to contaminated soil, with the feet, buttocks and thighs being the most commonly affected areas. ${ }^{1,3}$ Adult hookworms live in the intestines of dogs and cats, shedding eggs in feces that hatch and mature into larvae that can remain infective for months in the soil. ${ }^{2}$ Humans are typically deadend hosts, as the larvae wander aimlessly in the skin and

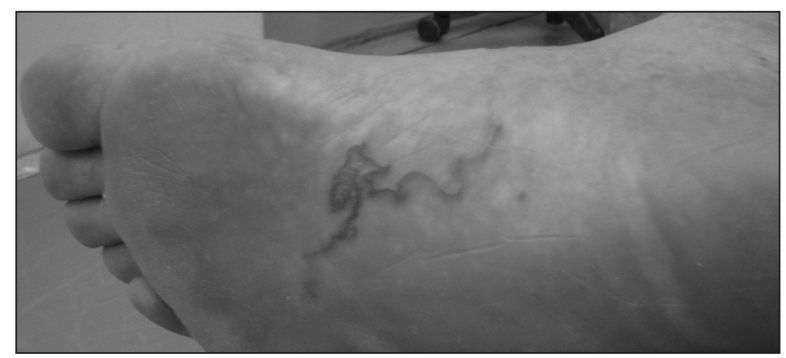

Fig. 1. Expanding pruritic lesion on the sole of the right foot of a 58-year-old man. cannot gain access to the lymphatic or venous systems to reach the intestines and complete their life-cycle. ${ }^{3}$

Intense pruritis and occasionally pain are the most frequently reported symptoms. Laboratory testing and skin biopsy are of little benefit. Although CLM is largely a selflimiting disease, $8 \%$ of patients get a bacterial superinfection, and $81 \%$ of patients experience disturbed sleep. ${ }^{1,2}$ Treatment with antihelminthic agents such as single-dose ivermectin or a 3-day course of albendazole has cure rates of $92 \%-100 \%$. ${ }^{1}$ Topical thiabendazole is also effective, but less desirable because of issues with patient compliance. ${ }^{2}$ During times of war, skin disorders are a common reason for outpatient visits by soldiers and lost combat days. ${ }^{4}$ Outbreaks of CLM in soldiers tend to be from tourist behaviour, not military activities. ${ }^{5}$

Competing interests: None declared.

Keywords: hookworm, CLM, cutaneous larva migrans, creeping eruption, tropical medicine, military medicine

\section{REFERENCES}

1. Hochedez P, Caumes E. Hookworm related cutaneous larva migrans. J Travel Med 2007;14:326-33.

2. Heukelbach J, Feldmeier H. Epidemiological and clinical characteristics of hookworm related cutaneous larva migrans. Lancet Infect Dis 2008;8:302-9.

3. Heukelbach J, Hengge UR. Bed bugs, leeches and hookworm larvae in the skin. Clin Dermatol 2009;27:285-90.

4. Jappe U. Unusual skin infections in military personnel. Clin Dermatol 2002;20:425-34.

5. Green AD, Mason C, Spragg PM. Outbreak of cutaneous larva migrans among British military personnel in Belize. J Travel Med 2001;8:267-9.

Correspondence to: Dr. Eric Tomich, 3851 Roger Brooke Dr., Department of Emergency Medicine, Brooke Army Medical Center, San Antonio TX 78234; eric.tomich@us.army.mil

From the Emergency Department, 10th Combat Support Hospital, Tallil, Iraq (origin of case), and the Department of Emergency Medicine, Madigan Army Medical Center, Tacoma, Wash. (hospital affiliation)

The opinions or assertions contained herein are the private views of the authors and not to be construed as official or reflecting the views of the Department of the Army, the Department of Defense or the US Government.

Submitted Oct. 27, 2009; Accepted Oct. 28, 2009

This article has been peer reviewed.

CJEM 2010;12(5):446 